\title{
GROUP RINGS, MATRIX RINGS, AND POLYNOMIAL IDENTITIES
}

\author{
BY
}

\section{ELIZABETH BERMAN}

\begin{abstract}
This paper studies the question, if $R$ is a ring satisfying a polynomial identity, what polynomial identities are satisfied by group rings and matrix rings over $R$ ? Theorem 2.6. If $R$ is an algebra over a field with at least $q$ elements, and $R$ satisfies $x^{q}=0$, and $G$ is a group with an abelian subgroup of index $k$, then the group ring $R(G)$ satisfies $x^{t}=0$, where $t=q k^{2}+2$. Theorem 3.2. If $R$ is a ring satisfying a standard identity, and $G$ is a finite group, then $R(G)$ satisfies a standard identity. Theorem 3.4. If $R$ is an algebra over a field, and $R$ satisfies a standard identity, then the $k-b y-k$ matrix ring $R_{k}$ satisfies a standard identity. Each theorem specifies the degree of the polynomial identity.
\end{abstract}

1. Introduction. We summarize results on matrix rings over polynomial identity rings. Let $[b]$ denote the largest integer in $b$. Suppose that $R$ is a ring satisfying a homogeneous polynomial identity with coefficients in the centroid, at least one coefficient of 1 , and degree $d$. Then for all $k, R_{k}$ satisfies some power of the standard identity of degree $2 k[d / 2]^{2}[6$, Theorem 1$]$, [2, Theorem 8$]$.

The unitary polynomial of degree $q$ is

$$
\sum_{f \in S_{q}} x_{f(1)} \cdots x_{f(q)}
$$

where the sum is over all permutations $f$ in $S_{q}$, the symmetric group on $q$ letters. The unitary identity results from setting the polynomial equal to 0 . If $R$ is a ring satisfying the unitary identity of degree $q$, then $R_{k}$ satisfies the unitary identity of degree $q k^{2}+1[2$, Theorem 1].

The docile polynomial of degree $q$ is

$$
\sum_{f}(-1)^{f} x_{f(1)} \cdots x_{f(q)} \text {, }
$$

where the sum is over all permutations $f$ in $S_{q}$ sending even integers into even integers. If $R$ is a ring satisfying the docile identity of degree $2 q$, then $R_{k}$ satisfies the standard identity of degree $2 q^{2} k^{2}+1$ [2, Theorem 2].

The docile product polynomial of degree $q, p$ is

$$
\prod_{i=1}^{p} D\left(x_{i 1}, \cdots, x_{i q}\right) u_{i},
$$

Received by the editors August 5, 1971. $20 \mathrm{C} 05$.

AMS (MOS) subject classifications (1970). Primary 16A22, 16A26, 16A38, 16A42,

Key words and phrases. Group rings, matrix rings, polynomial identities, standard identity, bounded nil rings. 
where $D$ is the docile polynomial of degree $q$, and the $x$ 's and $u$ 's are noncommuting variables. If $R$ is a ring satisfying the docile product polynomial identity of degree $2 q, p$, then $R_{k}$ satisfies a product of $p$ standard identities, each of degree $2 q^{2} k^{2}+1$ [1, Theorem 2.2].

If $R$ is an algebra over a field with at least $q$ elements, and $R$ satisfies all the homogeneous components of $\left(\sum_{i=1}^{q} x_{i}\right)^{q}$, then $R_{k}$ satisfies $x^{p}=0$, with $p=q k^{2}+1[1$, Theorem 1.2].

The next section uses these results on matrix rings to prove theorems on group rings. $\$ 3$ is independent of the preceding material and contains the main results of the paper: If $R$ is a ring satisfying the standard identity and $G$ is a finite group, then $R(G)$ satisfies a standard identity. A similar theorem for matrix rings is a corollary.

2. Various polynomial identities on group rings. We know that if $K$ is a field and $G$ a group with an abelian subgroup of finite index $k$, then $K(G)$ satisfies the standard identity of degree $k^{2}+1[6$, Theorem 4.2]. The proof below is similar.

Theorem 2.1. Suppose that $R$ is a ring and $G$ a group with subgroup $A$ of finite index $k$. Let $E$ be the group ring $R(A)$, and let $I$ be the set of all $r$ in $R$ such that $R r=0$. Then there is a bomomorphism of $R(G)$ into $E_{k}$ with kernel $I(G)$.

Proof. Let $y_{1}, \cdots, y_{k}$ be a set of right coset representatives of $A$ in $G$. Then $R(G)$ is a free left module over $E$ with basis $y_{1}, \ldots, y_{k}$. Let $T$ be the ring of linear transformations of this module. If $w \in R(G)$, let $T_{w}$ be the function on the module sending $y$ into $y w$. Then the map $w \rightarrow T_{w}$ is a homomorphism from the ring $R(G)$ into $T$ with kernel $I(G)$. Since $T$ is isomorphic to $E_{k}$, the theorem follows.

Corollary 2.2. Suppose that $R$ is a ring with unity and $G$ is a group with an abelian subgroup $A$ of finite index $k$. Then any bomogeneous multilinear polynomial identity satisfied by $R$ is also satisfied by $R(A)$, and any polynomial identity satisfied by $[R(A)]_{k}$ is also satisfied by $R(G)$.

Proof. If $R$ contains unity, $I=\{0\}$.

Remark. If $R$ is a ring without unity, it may be imbedded in a ring with unity, as is well known. If $R$ satisfies the docile identity, the docile product identity, or some power of the standard identity, then so does the new ring. Thus we get the following corollaries of three matrix ring theorems from the introduction:

2.3. If $R$ is a ring satisfying the docile identity of degree $2 q$, and $G$ is a group with abelian subgroup of index $k$, the $R(G)$ satisfies the standard identity of degree $2 q^{2} k^{2}+1$. 
2.4. If $R$ is a ring satisfying the docile product identity of degree $2 q, p$, and $G$ is a group with an abelian subgroup of index $k$, then $R(G)$ satisfies a product of $p$ standard identities, each of degree $2 q^{2} k^{2}+1$.

2.5. If $R$ is a ring satisying a bomogeneous polynomial identity with coefficients in the centroid, at least one coefficient of 1 , and $G$ is a group with an abelian subgroup of index $k$, then $R(G)$ satisfies some power of a standard identity.

For the unitary identity we prove the following:

Theorem 2.6. If $R$ is a ring satisfying the unitary identity of degree $q$, and $G$ is a group with an abelian subgroup of finite index $k$, then $R(G)$ satisfies $x_{0} U\left(x_{1}, \cdots, x_{p}\right)=0$, where $U$ is the unitary polynomial, $p=q k^{2}+1$, and the $x$ 's are noncommuting variables.

Proof. Let $E$ and $I$ be as in Theorem 2.1. There is a homomorphism from $R(G)$ into $E_{k}$ with kernel $I(G)$. Now $E_{k}$ satisfies $U\left(x_{1}, \cdots, x_{p}\right)=0$. Thus if $w_{1}, \cdots, w_{p}$ are $p$ elements of $R(G)$, then $U\left(w_{1}, \cdots, w_{p}\right) \in I(G)$. Since $I$ is the right annihilator of $R$, the theorem follows.

Theorem 2.7. If $R$ is an algebra over a field with at least $q$ elements, and $R$ satisfies $x^{q}=0$, and $G$ is a group with an abelian subgroup of index $k$, then $R(G)$ satisfies $x^{t}=0$, where $t=q k^{2}+2$.

Proof. The polynomial $\left(\sum_{i=1}^{q} x_{i}\right)^{q}$ vanishes on $R$. Using a Vandermonde determinant, we see that every homogeneous component of this polynomial vanishes on $R$ and hence also on $R(A)$. Thus $[R(A)]_{k}$ satisfies $x^{p}=0$, with $p=q k^{2}+1$. Thus if $w \in R(G), w^{p} \in I(G)$. Hence $w^{p+1}=0$.

3. The standard identity.

Definition. Suppose that $G$ is a semigroup and $\left\{g_{1}, \cdots, g_{q}\right\}$ is a sequence of $q$ elements of $G$. To parenthesize the sequence into $j$ clumps is to insert $j$ pairs of adjacent, nonoverlapping parentheses. The subsequence within one pair of parentheses constitute a clump. It is odd or even, depending on whether there is an odd or even number of elements.

In the example below we parenthesize a sequence into 3 clumps:

$$
\left\{g_{1}, g_{2},\left(g_{3}, g_{4},\right)\left(g_{5},\right)\left(g_{6}, g_{7},\right) g_{8}\right\} \text {. }
$$

The product of the elements within a clump is the value of the clump. If $C_{1}$, $\cdots, C_{k}$ are $k$ clumps, then the value of $C_{1} \cdots C_{k}$ is the product of the values of these clumps in the stated order.

Lemma 3.1. Suppose that $G$ is a group with unity $e$. Let $j$ and $n$ be positive integers, with $p=\sum_{i=0}^{n-1} j^{i}$. Let $S=\left\{g_{1}, \cdots, g_{p}\right\}$ be a sequence of $p$ 
elements of $G$. Then $S$ may be parenthesized into clumps such that at least one of the following conditions holds:

1. There are $n$ consecutive clumps $C_{1}, \ldots, C_{n}$ such that $C_{1}$ begins with $g_{1}$, and the values of $C_{1}, C_{1} C_{2}, \cdots, C_{1} C_{2} \cdots C_{n}$ are all distinct.

2. There are $j$ consecutive clumps each of value $e$.

Proof. Induct on $n$. If $n=1$, then $p=1$. If $S$ is a sequence composed of a solitary element $g_{1}$, then we form one clump consisting of $g_{1}$, and condition 1 holds.

Assume that the lemma is true for $n$. Let $p=\sum_{i=0}^{n-1} j^{i}$, and let $q=\sum_{i=0}^{n} j^{i}=$ $j p+1$.

Suppose that $\left\{g_{1}, \cdots, g_{q}\right\}$ is a sequence of $q$ elements of $G$. Let $S^{\prime}=$ $\left\{g_{2}, \cdots, g_{p+1}\right\}$. If $S^{\prime}$ has $j$ consecutive clumps of value $e$, then we are done. If not, then by induction hypothesis we can form $n$ consecutive clumps $C_{2}, \ldots, C_{n+1}$ in $S^{\prime}$ such that $C_{2}$ begins with $g_{2}$, and the values of $C_{2}, \cdots, C_{2} \cdots C_{n+1}$ are all distinct. Form a new clump $C_{1}$ consisting of $g_{1}$.

Case 1. The values of $C_{1}, C_{1} C_{2}, \ldots, C_{1} \cdots C_{n+1}$ are all distinct. Then conclusion 1 holds.

Case 2. The values are not all distinct. Then there exist positive integers $k$ and $r$ such that $k+r \leq n+1$ and $C_{1} \ldots C_{k}=C_{1} \ldots C_{k+r}$. If $k>1$, then we cancel the value of $C_{1}$ and obtain $C_{2} \cdots C_{k}=C_{2} \cdots C_{k+r^{*}}$ But this is impossible. Hence $k=1$, and

$$
C_{1}=C_{1} \cdots C_{1+r}
$$

Thus $e=C_{2} \cdots C_{1+r}$.

Combine the clumps $C_{2}, \ldots, C_{1+r}$ to form a new clump $D_{1}$ of value $e$. Let $g_{m}$ be the entry in the sequence following $D_{1}$. Continue the argument as before with the next $p$ entries, starting with $g_{m}$. If we do not finish then, we get a second clump $D_{2}$ of value $e$. Since there are $j p$ elements after $g_{1}$, we will get $j$ clumps $D_{1}, \cdots, D_{j}$, each of value $e$, if we do not finish earlier.

Theorem 3.1. Suppose that $n$ and $j$ are positive integers, $G$ is a group of order $n>1$, and $q=\left(j^{n}-1\right) /(j-1)$. Then any sequence of $q$ elements of $G$ can be parenthesized into $j$ clumps, each of value $e$.

Proaf. By the formula for the sum of a geometric progression $q=\sum_{i=0}^{n} j^{i}$. If the theorem is false, then $G$ has at least $n+1$ elements.

Lemma 3.2. If $\left\{g_{1}, \cdots, g_{q}\right\}$ is a sequence of elements of a set, and one parent hesizes them into $j$ odd clumps, and permutes the clumps with some permutation $f$ in $S_{j}$, then the resulting permutation of the original $q$ elements bas the same parity as $f[2$, Corollary to Lemma 4]. 
Lemma 3.3. Suppose that $n$ and $k$ are positive integers, and $A$ and $B$ are sets, $A$ containing at least $n(k-1)+1$ elements, $B$ containing exactly $n$ elements. Let $b$ be a function from $A$ into $B$. Then at least $k$ elements of $A$ bave the same image in $B$ [2, Lemma 1].

Lemma 3.4. Suppose that $A$ and $B$ are finite nonempty sets, $|A|=m$, and $|B|=n$. Let $b$ be a function from $A$ into $B$. Let $[j]$ be the largest integer in $j$, and let $k=[(m+n-1) / n]$. Then at least $k$ elements of $A$ bave the same image in $B$.

Proof. Since $k \leq(m+n-1) / n$, we have $n k \leq m+n-1$, and $n(k-1)+1$ $\leq m$. The previous lemma now applies.

Lemma 3.5. Suppose that $G$ is a group of order $n \geq 2$, and $p$ is a positive integer. Let $t=n(p-1)+2$. Let $q=\left(t^{n}-1\right) /(t-1)$. Then any sequence of $q$ elements in $G$ can be parenthesized into $2 p$ odd clumps whose values commute with each other.

Proof. By Theorem 3.1, the sequence can be parenthesized into $t$ clumps, each of value $e$. Let $E$ be the number of even clumps, and let $D$ be the number of odd clumps. Let $k=[(E+n-1) / n]$.

Let $A$ be the set of even clumps. If $A$ is nonempty, define a function from $A$ into $G$, letting the image of each even clump be its initial element. By Lemma 3.4 , since $|G|=n$, at least $k$ even clumps have the same initial element $g$. Choose the first $k$ such clumps; call them $\left\{C_{1}, \cdots, C_{k}\right\}=C$.

If $A$ is empty, $k=0$. Let $C$ be the empty set.

Let $J$ be the union of $C$ with all the odd clumps. Create new clumps as follows: Let $E_{1}$ be the first clump in $J$. If $E_{1}$ is odd, form the new clump $F_{1}$ by combining $E_{1}$ with all even clumps to the right, if any, up to the next clump from $J$. Then $F_{1}$ is odd, of value $e$.

If $E_{1}$ is even, form two new clumps $F_{1}$ and $F_{2}$ : let $F_{1}$ consist of the single element $g$ that begins $E_{1}$. Obviously, $F_{1}$ is odd, of value $g$. Let $F_{2}$ consist of all subsequent elements, up to the next clump from $J$. Now $E_{1}$, with $g$ deleted, is odd, of value $g^{-1}$. All following clumps not in $J$ are even, of value $e$. Thus $F_{2}$ is odd, of value $g^{-1}$.

Continuing in this manner, we form $2 k+D$ new clumps, adjacent, odd, and of value either $g, g^{-1}$, or $e$. We next show that $2 k+D \geq 2 p$.

Case 1. Suppose that $D=0$ or 1 . Now $E=t-D=n(p-1)+2-D$. Thus

$$
k=\left[\frac{E+n-1}{n}\right]=\left[\frac{n p+1-D}{n}\right] \geq \frac{n p+1-1}{n}=p \text {. }
$$

Hence $2 k+D \geq 2 p$. 
Case 2. Suppose that $D \geq 2$. Then

$$
\begin{aligned}
2 k+D & =2\left[\frac{n p+1-D}{n}\right]+D>2\left(\frac{n p+1-D}{n}-1\right)+D \\
& =2 \frac{n p+1}{n}-2+D\left(1-\frac{2}{n}\right) \geq 2 \frac{n p+1}{n}-2+2\left(1-\frac{2}{n}\right) \\
& =2 p-\frac{2}{n} \geq 2 p-1 .
\end{aligned}
$$

Since $2 k+D$ is an integer, $2 k+D \geq 2 p$.

Note. The next theorem hypothesizes that $R$ satisfies a standard identity of even degree. Leron and Vapne mention that a ring with unity satisfying a standard identity of degree $2 p+1$ also satisfies the standard identity of degree $2 p$ [4, p. 130].

Theorem 3.2. If $R$ is a ring satisfying the standard identity of degree $2 p$, and $G$ is a group of order $n>1$, and $q$ is the integer defined in Lemma 3.4, then $R(G)$ satisfies the standard identity of degree $q$.

Proof. Let $P\left(x_{1}, \cdots, x_{q}\right)=0$ be the standard identity of degree $q$. It suffices to prove that $P$ vanishes on elements of $R(G)$ of form $r g$, where $r \in R$, and $g \in G$. Let $r_{1} g_{1}, \cdots, r_{q} g_{q}$ be $q$ such elements, and let $f$ be any permutation in $S_{q}$. Let $S$ be the sequence in $G\left\{g_{f(1)}, \cdots, g_{f(q)}\right\}$. Then $S$ can be parenthesized into $2 p$ odd clumps whose values commute with each other. Consider $f$ as a sequence: $\{f(1), \ldots, f(q)\}$, and parenthesize $f$ in the same manner as $S$. We can partition $S_{q}$ into disjoint subsets, such that if $f$ and $f^{\prime}$ are in the same subset, they satisfy the following relations:

1. The integers before the first clump are the same integers in the same order.

2. The integers after the last clump are the same integers in the same order.

3. The $2 p$ odd clumps are the same, but in any order. Now

$$
P\left(r_{1} g_{1}, \cdots, r_{q} g_{q}\right)=\sum_{f \in S_{q}}(-1)^{f} r_{f(1)^{g}} g_{f(1)} \cdots r_{f(q)} g_{f(q)} .
$$

Let $T$ be one of the partition subsets defined above. Let $f^{\prime} \in T$, and let $g=g_{f^{\prime}(1)} \cdots g_{f^{\prime}(q)}$. Then

$$
\sum_{f \in T}(-1)^{f_{r}(1)} g_{f(1)} \cdots r_{f(q)} g_{f(q)}=\left[\sum_{f \in T}(-1)^{f_{r(1)}} \cdots r_{f(q)}\right] g .
$$

For each $f \in T$, we parenthesize the sequence $\left\{r_{f(1)}, \cdots, r_{f(q)}\right\}$ in the same manner as $S$, forming $2 p$ odd clumps. The expression within brackets above is the product of the following factors 
1. The product of all the r's before the first clump.

2. Polynomial $P$, evaluated on the $2 p$ odd clumps.

3. The product of all $r$ 's after the last clump.

Since the second factor is 0 , the theorem follows.

Theorem 3.3. Suppose that $R$ is a ring satisfying the standard identity of degree $2 p$. Let $k$ be a positive integer, and let $n=2^{k} k$ !. Let $q$ be as in Lemma 3.4. Suppose that $2^{q}$ does not annibilate $R$. Then $R_{k}$ satisfies $2^{q} P\left(x_{1}, \cdots, x_{q}\right)=0$, where $P$ is the standard polynomial.

Proof. As is well known, $R_{k}$ is isomorphic to $R \otimes Z_{k}$, where $Z$ is the ring of integers. Let $B$ be the subset of $Z_{k}$ of nonsingular matrices in which each row consists entirely of zeroes, except for a single entry of either 1 or -1 . Under matrix multiplication, $G$ is a group of order $2^{k} k$ !.

If $r \in R$, and $g \in G$, let $f(r g)=r \otimes g \in R \otimes Z_{k}$. Then $f$ can be extended to get a ring homomorphism of $R(G)$ into $R \otimes Z_{k}$. Suppose that $e_{i j}$ is the matrix unit in $Z_{k}$ with 1 in row $i$, column $j$. Let $w$ and $z$ be elements of $G$ such that every entry in $w$ is the negative of the corresponding entry in $z$, except that each has 1 in row $i$, column $j$. Then $2 e_{i j}=w+z$. Thus if $r \in R$, and $u$ is a matrix unit in $Z_{k}$, then $r \otimes 2 u$ lies in the image of the homomorphism.

If $r_{1}, \cdots, r_{q} \in R$, and $u_{1}, \cdots, u_{q}$ are matrix units, then

$$
2^{q} P\left(r_{1} \otimes u_{1}, \cdots, r_{q} \otimes u_{q}\right)=P\left(r_{1} \otimes 2 u_{1}, \cdots, r_{q} \otimes 2 u_{q}\right)=0 .
$$

Hence $2^{q} P$ vanishes on $R_{k}$.

Theorem 3.4. Hypothesis: Let $R$ be an algebra over a field F. Suppose that $R$ satisfies the standard identity of degree $2 p$. Let $k$ be a positive integer, and let $q$ be as in Lemma 3.4.

Conclusion: If $F$ has characteristic 2 , then $R_{k}$ satisfies the standard identity of degree $2 p k^{2}+1$. If $F$ bas another characteristic, then $R_{k}$ satisfies the standard identity of degree $q$.

Proof. The first statement is the Corollary to Theorem 1 from [2]. The second is a corollary of Theorem 3.3.

\section{REFERENCES}

1. Elizabeth Berman, Matrix rings over polynomial identity rings, Trans. Amer. Math. Soc. 172 (1972), 231-239.

2. — Tensor products of polynomial identity algebras, Trans. Amer. Math. Soc. 156 (1971), 259-271. MR 43 \#278.

3. Nathan Jacobson, Structure of rings, rev. ed., Amer. Math Soc. Colloq. Publ., vol. 37, Amer. Math. Soc., Providence, R. I., 1964. MR $36 \# 5158$. 
4. Uri Leron and Amitai Vapne, Polynomial identities of related rings, Israel J. Math. 8 (1970), 127-137. MR 42 \#4589.

5. D. S. Passman, Linear identities in group rings, Pacific J. Math. 36 (1971), 457483.

6. C. Procesi and L. Small, Endomorphism rings of modules over PI-algebras, Math. Z. 106 (1968), 178-180. MR 38 \#2167.

DEPAR TMENT OF MATHEMATICS, ROCKHURST COLLEGE, KANSAS CITY, MISSOURI 64110 